\title{
Applying health belief model for the assessment of community knowledge, attitude and prevention practices following a dengue epidemic in a township in Selangor, Malaysia
}

\author{
Hidayatulfathi Othman ${ }^{1}$, Zul-Izzat Ikhwan Zaini ${ }^{1}$, Norhafizah Karim ${ }^{1}$, \\ Nor Azimah Abd Rashid ${ }^{1}$, Muhammad Badrul Hisham Abas ${ }^{1}$, Mazrura Sahani ${ }^{1}$, Rozita Hod ${ }^{2}$, \\ Faiz Daud $^{2}$, Saiful Azlan Nordin ${ }^{1}$, Nor Azwani Mohamed Nor ${ }^{1}$
}

\begin{abstract}
${ }^{1}$ Centre of Health and Applied Sciences, Faculty of Health Sciences, Universiti Kebangsaan Malaysia, Jalan Raja Muda Abdul Aziz, Kuala Lumpur, Malaysia

${ }^{2}$ Department of Public Health, Faculty of Medicine, Universiti Kebangsaan Malaysia, Jalan Yaacob Latif, Bandar Tun Razak, Cheras, Wilayah Persekutuan Kuala Lumpur, Malaysia
\end{abstract}

Received: 10 December 2018

Revised: 18 January 2019

Accepted: 19 January 2019

\section{*Correspondence: \\ Dr. Hidayatulfathi Othman, \\ E-mail: hida@ukm.edu.my}

Copyright: ( ) the author(s), publisher and licensee Medip Academy. This is an open-access article distributed under the terms of the Creative Commons Attribution Non-Commercial License, which permits unrestricted non-commercial use, distribution, and reproduction in any medium, provided the original work is properly cited.

\begin{abstract}
Background: Dengue fever is endemic in Malaysia and continues to be a public health concern. Selangor was the top hit and there is a paucity of information on knowledge, attitudes, and practices (KAP) of Bandar Baru Bangi residents regarding dengue infection. Therefore, the objective of this study is to describe dengue related knowledge, attitudes and practices (KAP) of residents of Bandar Baru Bangi.

Methods: A cross-sectional questionnaire survey of 152 residents from Section 3 and Section 8, Bandar Baru Bangi attending the dengue awareness events was conducted. The information on socio-demographic characteristics of the participants and their knowledge, attitude and practice on dengue fever was collected using a structured questionnaire. Results: The results showed that the residents only $52 \%$ had sufficient knowledge about the dengue vectors, signs, symptoms, and modes of transmission. However, approximately all the respondents considered dengue as serious but preventable disease to which they are vulnerable to. Television/radio was the predominant sources of information about dengue fever. Knowledge on dengue is associated with prevention practice. But the self-efficacy has a significant impact between the owner and tenant house $(\mathrm{p}=0.010, \mathrm{p}<0.05)$. Female has higher self-efficacy compare to male.

Conclusions: As conclusion, findings suggest that despite the residents of Bandar Baru Bangi have moderate knowledge level about dengue fever nevertheless; they still adopted the preventive measures suggested by the guidelines from Ministry of Health. Therefore, health program planners and practitioners need to identify why the dengue fever still rampant among the population.
\end{abstract}

Keywords: Dengue, Health belief model, KAP, Aedes, Dengue free community

\section{INTRODUCTION}

Dengue fever is one of the fastest growing infectious diseases in the world, with the World Health
Organization (WHO) estimating 390 million cases of dengue each year, with as many as two and a half billion people at risk from the disease. ${ }^{1}$ Asia bore $70 \%$ of this burden, and is characterized by large swathes of densely populated regions coinciding with very high suitability 
for disease transmission. ${ }^{2}$ Aedes mosquito is responsible to transmit for four vector-borne viruses; dengue, chikungunya, yellow fever and the latest is Zika. ${ }^{3}$

The year 2015 was characterized by large dengue outbreaks worldwide, with Malaysia exceeding 111000 cases of dengue, representing a $59.5 \%$ increase in numbers of cases to the previous year, respectively. ${ }^{1}$ In Malaysia, among the factors that caused the increase in cases identified dengue in the state is related to cleanliness unattended environments that contribute to Aedes breeding grounds such areas, construction sites, abandoned apartments projects were poorly and so on. Besides, it's also due to the attitude of people who are not clean up their environment and do not do activities search-and-destroy Aedes breeding grounds every week. ${ }^{4}$

Health education for dengue control has been effective in improving awareness regarding dengue. ${ }^{2,5-7}$ To plan for effective dengue control programs, it is important to assess the potential maximal effect of preventive behaviors in reducing the mosquito density especially after the place was fogged and sprayed with insecticide. The health authorities have taken all the control measures together with many health education programs following those dengue outbreak episodes. In order to explain the level of attitude of the community, we adopt the health belief model (HBM), a well-established theoretical approach, to address the problem of dengue cases in the area. $^{2}$

Despite the magnitude of problem, no documented evidence exists on the knowledge, attitude and prevention practices of the adult population in the hotspot areas regarding dengue fever. Given this void, the aim of our study was to assess the level of knowledge about dengue, its spread, symptoms and prevention among the population of Bandar Baru Bangi, a township that worst hit by dengue outbreaks in the end of 2014. We were also keen to find out the level of attitude and the preventive measures against dengue fever in the area.

\section{METHODS}

Study duration and location: A cross-sectional study survey was conducted in January 2016 at Bandar Baru Bangi, Selangor specifically at Section 8 (Bangi Prima) and Section 3 (Bangi Perdana). Total area for Section 8 is 28 hectares with a total of 640 double-storey houses whereas Section 3 (Bangi Perdana) covers 18 hectares with 600 double-storey houses in total. The distance between these two locations is approximately $3.13 \mathrm{~km}$. The facilities between these two locations are similar to one another with playground, near shop lot building, and listed as hotspot locality by the authorities. Bandar Baru Bangi is a tropical climate with a distinct monsoon season (April-October) and a dry season (NovemberMarch). The climate tends to get hot with the presence of El Nino phenomena at the end of 2015 continued until early 2016. The total dengue cases in Section 8 was 11 cases in 2014, increase to 42 cases in 2015 and up to 17 cases for the month of January and February 2016. On the other hand, Section 3 reported a total of 98 dengue cases for year 2014, increase to 147 cases in 2015 and 17 cases for January and February 2016.

Sampling procedure and survey: A purposive sampling method was used to select a sample of participants to represent the general community of Section 8 and 3, Bandar Baru Bangi. By using a sample size calculator $(\mathrm{G}$ power calculator), it was estimated that a minimum of 152 households should be sampled in order to gain a $80 \%$ confidence level, with a maximum allowable difference of 0.05 in detecting the KAP proportions in the population. A dengue awareness event was held at both localities separately and the survey was done reporting a total number of 152 respondents (80 respondents from Section 8 and 72 respondents from section 3 ).

A structured questionnaire on knowledge, attitude and practice of dengue control was prepared. It was printed in local language since and the questionnaire was divided into four sections; the first section covered the knowledge on Aedes and dengue consist of 11 questions, the second part also related to knowledge but on breeding sites and preventive measures comprised of 5 questions and the third section concerned with attitude consists of 30 questions build based on Health Belief Model (HBM) which covers six concepts which are severity, susceptibility, cues to action, self-efficacy, perceived barrier and perceived benefit. For scoring, a Likert scale 1 to 4 was given to each perception varying from very agree, agree, less agree and disagree. The last part related to practices with 12 questions in total. The overall reliability of the questionnaire was acceptable with Cronbach-alpha value of 0.763 . The study was approved by the Ethical Review Committee for Faculty of Health Sciences Department, National University of Malaysia (Ethical code no. UKM PPI/111/8/JEP-2016-393)

Data analysis: The data collected from the questionnaire was entered and analyzed using Statistical Package for Social Sciences (SPSS) version 23.0. All of the questions were analyzed and assessed individually using a scoring system. Each of the appropriate answer was given a point and was totaled for each section. Respondents were considered to have sufficient knowledge (43 questions), satisfactory attitude (30 questions) and have good preventive practices (10 questions) if they correctly answered $75 \%$ in each of the section of the questions. ${ }^{8}$

\section{RESULTS}

Overall, 152 residents agreed to participate with more than half of the respondent's was male $(75.0 \%, \mathrm{n}=114)$ as the targeted respondent was the head of households. $47.4 \%(n=72)$ of the respondent aged between $41-50$ years old and $52.0 \% \quad(n=78)$ were college graduates. Table 1 describes the socio-demographic of the respondents. The majority of our sample declared they 
never got dengue previously $(70.4 \%, \mathrm{n}=107)$. The main source of information was from the television/radio (89.8\%, $\mathrm{n}=168)$ followed by information from newspaper/magazine $(77.5 \%, \quad \mathrm{n}=145)$ and internet (73.3\%, n=137) (Figure 1). The most under-utilized source of information was from health personal and authorities $(60.4 \%, \mathrm{n}=113)$.

Table 1: Socio-demographic characteristics of the participants in the survey $(n=152)$.

\begin{tabular}{|c|c|c|c|}
\hline $\begin{array}{l}\text { S. } \\
\text { no. }\end{array}$ & Variables & $\mathbf{N}$ & $\%$ \\
\hline \multirow[t]{3}{*}{1} & Gender & & \\
\hline & Male & 114 & 75.0 \\
\hline & Female & 38 & 25.0 \\
\hline \multirow[t]{4}{*}{2} & Age & & \\
\hline & $18-30$ & 8 & 5.2 \\
\hline & $31-50$ & 23 & 15.2 \\
\hline & $>50$ & 121 & 79.6 \\
\hline \multirow[t]{4}{*}{3} & Marital status & & \\
\hline & Single & 8 & 5.3 \\
\hline & Married & 141 & 92.8 \\
\hline & Widower/widow & 3 & 2.0 \\
\hline \multirow[t]{7}{*}{4} & Educational attained & & \\
\hline & No formal education & 0 & 0 \\
\hline & Primary & 0 & 0 \\
\hline & Secondary & 21 & 14.0 \\
\hline & Diploma & 34 & 22.7 \\
\hline & Degree & 78 & 52.0 \\
\hline & Others & 17 & 11.3 \\
\hline \multirow[t]{3}{*}{5} & Residential property & & \\
\hline & Owner & 136 & 89.5 \\
\hline & Rent & 16 & 10.5 \\
\hline \multirow[t]{3}{*}{6} & History with dengue & & \\
\hline & Yes & 45 & 29.6 \\
\hline & No & 107 & 70.4 \\
\hline
\end{tabular}

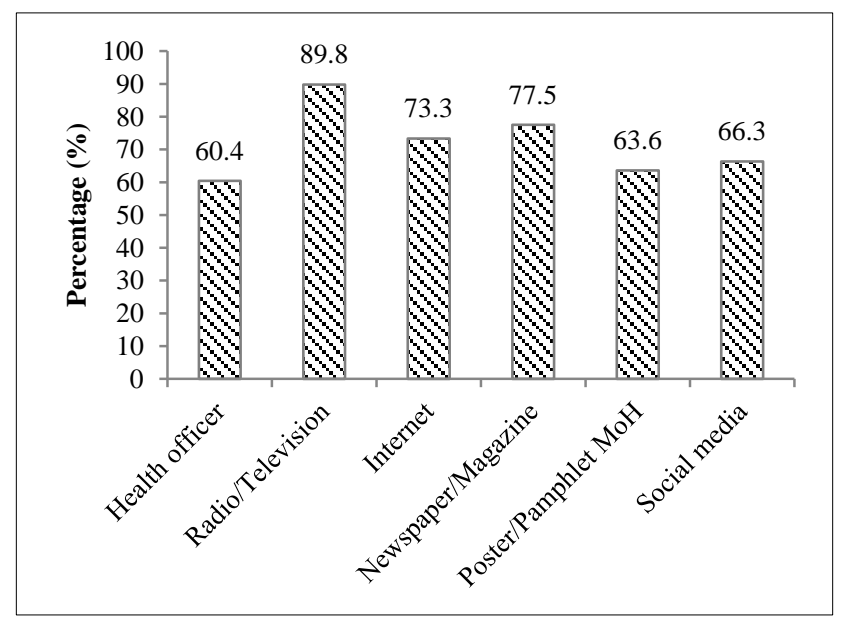

Figure 1: Sources of information received by the respondents $(n=152)$.

\section{Knowledge on dengue}

Data showing the extent of knowledge regarding vector breeding sites, mosquito bite time and preventive practices is presented in Table 2. Sufficient knowledge about dengue was found to be in $52.0 \%(\mathrm{n}=79)$ of the sample. $96.7 \%(n=147)$ respondents know that dengue is a serious threat in their areas. $80.3 \%(n=122)$ recognized that places that can contain water are the causes of dengue incidents in their areas. All 152 respondents believed that everyone was at risk of contracting dengue. The entire respondent except one respondent believed that dengue is dangerous. Out of those respondents who answered dengue is dangerous, $98.7 \%(n=150)$ believed it dangerous because it can cause death and there was no cure for dengue $42.8 \%$, $(n=65)$.

Most of the respondents have sufficient knowledge on the symptoms of dengue infection. We used a multiple response question to assess knowledge of the symptomatology of dengue; respondents reported the following as being most commonly associated with dengue: muscle pain $(90.1 \%, \mathrm{n}=137)$, fever $(88.8 \%$, $\mathrm{n}=135)$, skin $\operatorname{rash}(87.5 \%, \mathrm{n}=133)$, headaches $(73.0 \%$, $\mathrm{n}=111)$, nausea/vomiting $(55.9 \%, \mathrm{n}=85)$, bleeding $(46.1 \%, \mathrm{n}=70)$ and diarrhea $(28.3 \%, \mathrm{n}=43)$. When queried what they would do if they were infected with dengue, $81.6 \% \quad(n=124)$ of the respondents said they would immediately seek for treatment while $8.6 \%(n=13)$ said they would drink lots of water first. When asked about the causative agent for dengue, $74.3 \%(n=113)$ of the respondents answered Aedes mosquitoes. Only 23.0\% $(n=35)$ gave the correct answer, which is the dengue virus.

\section{Knowledge on Aedes mosquito}

The majority $(81.6 \%, \mathrm{n}=124)$ of the respondents know that Aedes mosquito is the carrier of the disease. When asked about the characteristics of the Aedes mosquito, most of the participants $(81.6 \%, \mathrm{n}=124)$ were aware that the Aedes mosquito has black and white stripes on its legs and body. However, 9 respondents (5.9\%) didn't know the characteristic of the Aedes mosquito. On the timing of the mosquito's biting habits, 100 (65.8\%) of the respondents said 'early morning and late evening', while $29(19.1 \%)$ respondents said 'late evening and dusk', while 19 (12.5\%) answered 'at dawn and early morning'. However, there are $4(2.6 \%)$ of the respondents who answered they didn't know the timing of the Aedes mosquito biting time.

When inquired, only $73(48 \%)$ respondents knew about the duration of Aedes mosquito life cycle from egg to adult. When probed further on the distance that these Aedes mosquito could fly on its own, 36.2\% ( $n=55)$ answered they didn't know. Only $25.7 \%(n=39)$ of the respondents correctly answered '200 meter'. 
Table 2: Knowledge on Aedes and dengue.

\begin{tabular}{|c|c|c|c|}
\hline S. no. & Variables & $\mathbf{N}$ & Percentage (\%) \\
\hline \multirow[t]{3}{*}{1} & Dengue is becoming a health problem in the area & & \\
\hline & Yes & 147 & 96.7 \\
\hline & No & 5 & 3.3 \\
\hline \multirow[t]{8}{*}{1.1} & Causes of dengue in the area & & \\
\hline & Unmanaged waste & 94 & 61.8 \\
\hline & Bushes around residential area & 100 & 65.8 \\
\hline & Empty houses/building & 92 & 60.5 \\
\hline & Abandoned car & 65 & 42.8 \\
\hline & Placed that can stored water examples pot liner etc. & 122 & 80.3 \\
\hline & Dengue infection from outside the residential area & 57 & 37.5 \\
\hline & Don't know & 3 & 2.0 \\
\hline \multirow[t]{5}{*}{2} & Who can be infected by dengue? & & \\
\hline & Children only & 0 & 0 \\
\hline & Adults only & 0 & 0 \\
\hline & Anyone & 152 & 100 \\
\hline & Don't know & 0 & 0 \\
\hline \multirow[t]{3}{*}{3} & Dengue is dangerous & & \\
\hline & Yes & 151 & 99.3 \\
\hline & No & 1 & 0.7 \\
\hline \multirow[t]{4}{*}{3.1} & Why? & & \\
\hline & Can caused death & 150 & 98.7 \\
\hline & No cure & 65 & 42.8 \\
\hline & Don't know & 1 & 0.7 \\
\hline \multirow[t]{9}{*}{4} & Sign and symptoms & & \\
\hline & Continuous fever & 135 & 88.8 \\
\hline & Skin rash & 133 & 87.5 \\
\hline & Muscle pain & 137 & 90.1 \\
\hline & Nausea/vomitting & 85 & 55.9 \\
\hline & Headache & 111 & 73.0 \\
\hline & Small bleeding at gum and nose & 70 & 46.1 \\
\hline & Diarrhea & 43 & 28.3 \\
\hline & Don't know & 1 & 0.7 \\
\hline \multirow[t]{9}{*}{5} & Treatment & & \\
\hline & Immediately see a doctor & 124 & 81.6 \\
\hline & Eat crab soup & 6 & 3.9 \\
\hline & Drink 100 plus (isotonic drinks) & 2 & 1.3 \\
\hline & Drink plain water & 13 & 8.6 \\
\hline & Drink papaya leaf juice & 3 & 2.0 \\
\hline & Drink coconut juice & 0 & 0.0 \\
\hline & Drink virgin coconut oil & 2 & 1.3 \\
\hline & Don't know & 2 & 1.3 \\
\hline \multirow[t]{9}{*}{6} & Causes of dengue & & \\
\hline & Biting of any mosquito & 2 & 1.3 \\
\hline & Biting of Aedes mosquito & 113 & 74.3 \\
\hline & Dengue virus & 35 & 23 \\
\hline & Rats & 0 & 0 \\
\hline & Wind & 0 & 0 \\
\hline & Contaminated water & 1 & 0.7 \\
\hline & Contaminated food & 0 & 0 \\
\hline & Don't know & 1 & 0.7 \\
\hline
\end{tabular}

Continued 


\begin{tabular}{|c|c|c|c|}
\hline & Variables & $\mathbf{N}$ & Percentage (\%) \\
\hline \multirow[t]{9}{*}{7} & Carrier for dengue & & \\
\hline & Biting of any mosquito & 3 & 2.0 \\
\hline & Biting of Aedes mosquito & 124 & 81.6 \\
\hline & Dengue virus & 22 & 14.5 \\
\hline & Rats & 0 & 0 \\
\hline & Wind & 0 & 0 \\
\hline & Contaminated water & 2 & 1.3 \\
\hline & Contaminated food & 0 & 0 \\
\hline & Don't know & 1 & 0.7 \\
\hline \multirow[t]{6}{*}{8} & Characteristics of the insect & & \\
\hline & Brown and hairy & 2 & 1.3 \\
\hline & Black and white stripes on the head & 17 & 11.2 \\
\hline & Shelled body with stripes on the wings & 0 & 0 \\
\hline & Black and white stripes on the body and legs & 124 & 81.6 \\
\hline & Don't know & 9 & 5.9 \\
\hline \multirow[t]{6}{*}{9} & Timing of the insect's biting habits & & \\
\hline & Dawn and early morning & 19 & 12.5 \\
\hline & Late evening and dusk & 29 & 19.1 \\
\hline & At night only & 0 & 0 \\
\hline & Early morning and late evening & 100 & 65.8 \\
\hline & Don't know & 4 & 2.6 \\
\hline \multirow[t]{5}{*}{10} & Life cycle of the insects from egg to adult & & \\
\hline & 7 days & 73 & 48.0 \\
\hline & 14 days & 21 & 13.8 \\
\hline & 21 days & 21 & 13.8 \\
\hline & Don't know & 37 & 24.3 \\
\hline \multirow[t]{6}{*}{11} & Distance for the insects to fly on its own & & \\
\hline & $50 \mathrm{~m}$ & 15 & 9.9 \\
\hline & $100 \mathrm{~m}$ & 16 & 10.5 \\
\hline & $200 \mathrm{~m}$ & 39 & 25.7 \\
\hline & $1 \mathrm{~km}$ & 27 & 17.8 \\
\hline & Don't know & 55 & 36.2 \\
\hline
\end{tabular}

Throw waste in tied plastic trash and tightly closed the trasch bin

Ensure water containers was tightly closed each time I used it

Do not keep damaged equipment that can stored water

Remove water inside container once a week

Participate in gotong-royong activities to prevent dengue outbreak

Allow fogging inside the house whenever the health team around

Brush and scrub water containers once a week

Check for mosquito breeding spot outside the house

Check for mosquito breeding spot inside the house

Use mosquito aerosol/spray everyday

Put larvicide to kill mosquito larvae in water storage

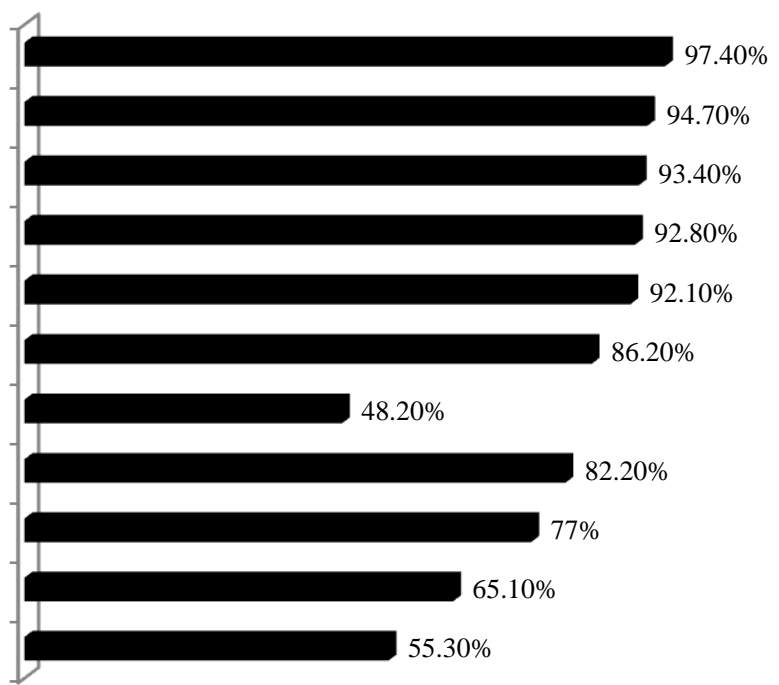

Figure 2: Prevention practices of the respondents in Bandar Baru Bangi, Selangor ( $n=152)$. 
Table 3: Knowledge on breeding spot and prevention.

\begin{tabular}{|c|c|c|c|}
\hline S. no. & Variables & $\mathbf{N}$ & $\%$ \\
\hline \multirow[t]{4}{*}{1} & What is the insects breeding spots & & \\
\hline & Clear water & 138 & 90.8 \\
\hline & Murky water & 12 & 7.9 \\
\hline & Don't know & 2 & 1.3 \\
\hline \multirow[t]{7}{*}{2} & Where is the potential breeding spots inside house & & \\
\hline & Pot liner & 135 & 88.8 \\
\hline & Kitchen cutlery tray & 116 & 76.3 \\
\hline & Toilet flush/tank & 111 & 73.0 \\
\hline & Cistern & 129 & 84.9 \\
\hline & Refrigerator tray & 123 & 80.9 \\
\hline & Don't know & 2 & 1.3 \\
\hline \multirow[t]{8}{*}{3} & Where is the potential breeding spots outside house & & \\
\hline & Clogged gutter/drain & 106 & 69.7 \\
\hline & Water storage container, jar & 110 & 72.4 \\
\hline & Unclosed cans and bottles (containers) & 134 & 88.2 \\
\hline & Solid waste that can stored water & 131 & 86.2 \\
\hline & Old tires that can stored water & 145 & 95.4 \\
\hline & Dark places & 36 & 23.7 \\
\hline & Don't know & 3 & 2.0 \\
\hline \multirow[t]{9}{*}{4} & How to prevent breeding spot & & \\
\hline & Allow fogging inside the house & 129 & 84.9 \\
\hline & Managed waste and recyclables properly & 115 & 75.7 \\
\hline & Used mosquito aerosol spray & 96 & 63.2 \\
\hline & Install mosquito nets at house windows & 96 & 63.2 \\
\hline & Prevent stagnant water & 130 & 85.5 \\
\hline & Always closed containers that can stored water & 126 & 82.9 \\
\hline & Eliminate mosquito breeding spots weekly & 137 & 90.1 \\
\hline & Don't know & 2 & 1.3 \\
\hline \multirow[t]{6}{*}{5} & What are the sentence imposed if breeding spot detected at your house area & & \\
\hline & Compound/Fine & 116 & 76.3 \\
\hline & Prison & 2 & 1.3 \\
\hline & Counsel & 8 & 5.3 \\
\hline & Clean the residential area & 19 & 12.5 \\
\hline & Don't know & 7 & 4.6 \\
\hline
\end{tabular}

Most of the respondents have sufficient knowledge on the breeding spots and prevention of the Aedes mosquito as shown in Table 3. About $90.8 \%(n=138)$ recognized that Aedes mosquito breeds in clean standing water. The most important potential breeding spots inside the houses were reported as pot liners $(88.8 \%, \mathrm{n}=135)$, cistern $(84.9 \%$, $\mathrm{n}=129)$, refrigerator tray $(80.9 \%, \mathrm{n}=123)$, kitchen cutlery tray $(76.3 \%, \mathrm{n}=116)$ and toilet flush/tank $(73.0 \%, \mathrm{n}=111)$. The most common breeding place outside the premises recognized was old tires $(95.4 \%, \mathrm{n}=145)$, unclosed can, bottles and containers $(84.2 \%, \mathrm{n}=134)$, solid waste $(86.2 \%, \mathrm{n}=131)$, water storage containers $(72.4 \%, \mathrm{n}=110)$ and clogged gutter/drain $(69.7 \%, \mathrm{n}=106$, respectively).

When asked on how to prevent breeding spots, most of the respondents had knowledge on ways to prevent Aedes breeding (98.7\%, $\mathrm{n}=150)$. Eliminate mosquito breeding spots weekly is on the top of the list of preventive measures $(90.1 \%, \mathrm{n}=137)$ followed by allowing the health authorities to spray the insecticide inside their premises $(84.9 \%, \mathrm{n}=129)$ and also prevent stagnant water $(85.5 \%, \mathrm{n}=130)$. One of the interesting fact is the respondents deliberated by the properly managed waste and recyclables, could be one of the preventive practice $(75.7 \%, \mathrm{n}=115)$. The least preventive practices are the usage of the mosquito aerosol spray $(63.2 \%, \mathrm{n}=96)$ and installing the mosquito nets at the window sills $(63.2 \%$, $n=96) .116(76.3 \%)$ of the respondents were aware that the punishment imposed if breeding spot detected in their residence area and 2 respondents know that they could get imprison if breeding spots are found in their premises as it is an infringement of the law. However, there are 7 (4.6\%) respondents didn't know what are the sentences imposed on them for breeding the Aedes larvae. 
Table 4: Attitude scoring of the respondents based on health belief model.

\begin{tabular}{|c|c|c|c|c|c|c|c|c|c|}
\hline & $\mathbf{N}$ & $\%$ & & Statements & $\begin{array}{l}\text { Strongly } \\
\text { agree }(\%)\end{array}$ & $\begin{array}{l}\text { Agree } \\
(\%)\end{array}$ & $\begin{array}{l}\text { Disagree } \\
(\%)\end{array}$ & $\begin{array}{l}\text { Strongly } \\
\text { disagree } \\
(\%)\end{array}$ & KMO \\
\hline \multicolumn{10}{|c|}{ Perceived severity of the disease } \\
\hline $\begin{array}{l}\text { Unsatisfactory } \\
\text { attitude }\end{array}$ & 45 & 29.6 & 1 & $\begin{array}{l}\text { I feel that dengue } \\
\text { fever is very } \\
\text { dangerous }\end{array}$ & $131(86.2)$ & $21(13.8)$ & 0 & 0 & \multirow{5}{*}{0.612} \\
\hline \multirow[t]{4}{*}{$\begin{array}{l}\text { Satisfactory } \\
\text { attitude }\end{array}$} & 107 & 70.4 & 2 & $\begin{array}{l}\text { I do not think dengue } \\
\text { fever can caused death }\end{array}$ & $8(5.3)$ & $5(3.3)$ & $25(16.4)$ & $114(75.0)$ & \\
\hline & & & 8 & $\begin{array}{l}\text { Dengue fever can } \\
\text { occur without } \\
\text { presences of skin } \\
\text { rashes }\end{array}$ & $52(34.2)$ & $83(54.6)$ & $13(8.6)$ & $4(2.6)$ & \\
\hline & & & 9 & $\begin{array}{l}\text { Continuous fever } \\
\text { worries me }\end{array}$ & $99(65.1)$ & $50(32.9)$ & $2(1.3)$ & $1(0.7)$ & \\
\hline & & & 13 & $\begin{array}{l}\text { Even though there are } \\
\text { various medical } \\
\text { facilities to treat me, } \\
\text { I'm still afraid of } \\
\text { dengue fever }\end{array}$ & $77(50.7)$ & $71(46.7)$ & $3(2.0)$ & $1(0.7)$ & \\
\hline \multicolumn{10}{|c|}{ Susceptibility towards the disease } \\
\hline $\begin{array}{l}\text { Unsatisfactory } \\
\text { attitude }\end{array}$ & 129 & 84.9 & 3 & $\begin{array}{l}\text { I was very worried } \\
\text { when get bitten by a } \\
\text { mosquito }\end{array}$ & $58(38.2)$ & $81(53.3)$ & $11(7.2)$ & $2(1.3)$ & \multirow{5}{*}{0.58} \\
\hline \multirow[t]{4}{*}{$\begin{array}{l}\text { Satisfactory } \\
\text { attitude }\end{array}$} & 23 & 15.1 & 10 & $\begin{array}{l}\text { I am not worry if there } \\
\text { is a standing of water } \\
\text { that is unnecessary } \\
\text { outside my house }\end{array}$ & $4(2.6)$ & $10(6.6)$ & $20(13.2)$ & $118(77.6)$ & \\
\hline & & & 11 & $\begin{array}{l}\text { I can be infected with } \\
\text { dengue fever }\end{array}$ & $88(57.9)$ & $57(37.5)$ & $4(2.6)$ & $3(2.0)$ & \\
\hline & & & 12 & $\begin{array}{l}\text { If I'm healthy, I will } \\
\text { not be infected with } \\
\text { dengue fever }\end{array}$ & $10(6.6)$ & $17(11.2)$ & 44 (28.9) & $81(53.3)$ & \\
\hline & & & 14 & $\begin{array}{l}\text { I was often bitten by } \\
\text { mosquitoes but never } \\
\text { fall sick. I thought I } \\
\text { will not be infected } \\
\text { with dengue fever }\end{array}$ & $7(4.6)$ & $10(6.6)$ & $46(30.3)$ & 89 (58.6) & \\
\hline \multicolumn{10}{|c|}{ Cues to take action against the vector } \\
\hline $\begin{array}{l}\text { Unsatisfactory } \\
\text { attitude }\end{array}$ & 28 & 18.4 & 4 & $\begin{array}{l}\text { I take precautions if } \\
\text { my area is declared a } \\
\text { hotspot area }\end{array}$ & $112(73.7)$ & $\begin{array}{l}40 \\
(26.3)\end{array}$ & 0 & 0 & \multirow{4}{*}{0.757} \\
\hline \multirow[t]{3}{*}{$\begin{array}{l}\text { Satisfactory } \\
\text { attitude }\end{array}$} & 124 & 81.6 & 5 & $\begin{array}{l}\text { I was often reminded } \\
\text { to take implementation } \\
\text { activities to control } \\
\text { dengue fever }\end{array}$ & $78(51.3)$ & $\begin{array}{l}67 \\
(44.1)\end{array}$ & $6(3.9)$ & $1(0.7)$ & \\
\hline & & & 6 & $\begin{array}{l}\text { People around me do } \\
\text { not carry out activities } \\
\text { to control dengue } \\
\text { fever }\end{array}$ & $7(4.6)$ & $\begin{array}{l}36 \\
(23.7)\end{array}$ & $77(50.7)$ & $32(21.1)$ & \\
\hline & & & 7 & $\begin{array}{l}\text { I will help my } \\
\text { neighbours eradicate } \\
\text { breeding spots if I see } \\
\text { them doing so }\end{array}$ & $75(49.3)$ & $\begin{array}{l}73 \\
(48.0)\end{array}$ & $3(2.0)$ & $1(0.7)$ & \\
\hline
\end{tabular}




\begin{tabular}{|c|c|c|c|c|c|c|c|c|c|}
\hline & $\mathbf{N}$ & $\%$ & & Statements & $\begin{array}{l}\text { Strongly } \\
\text { agree }(\%)\end{array}$ & $\begin{array}{l}\text { Agree } \\
(\%)\end{array}$ & $\begin{array}{l}\text { Disagree } \\
(\%)\end{array}$ & $\begin{array}{l}\text { Strongly } \\
\text { disagree } \\
(\%)\end{array}$ & KMO \\
\hline & & & 15 & $\begin{array}{l}\text { Announcements of } \\
\text { information about } \\
\text { dengue interest me to } \\
\text { take part in control/ } \\
\text { prevention of dengue }\end{array}$ & $59(38.8)$ & $\begin{array}{l}86 \\
(56.6)\end{array}$ & $6(3.9)$ & $1(0.7)$ & \\
\hline \multicolumn{10}{|c|}{ Self-efficacy (se) } \\
\hline $\begin{array}{l}\text { Unsatisfactory } \\
\text { attitude }\end{array}$ & 144 & 94.7 & 16 & $\begin{array}{l}\text { I will ensure there are } \\
\text { no breeding spots } \\
\text { around my house }\end{array}$ & $92(60.5)$ & $\begin{array}{l}59 \\
(38.8)\end{array}$ & 0 & $1(0.7)$ & \multirow{5}{*}{0.683} \\
\hline \multirow[t]{4}{*}{$\begin{array}{l}\text { Satisfactory } \\
\text { attitude }\end{array}$} & 8 & 5.3 & 19 & $\begin{array}{l}\text { I felt that larvae } \\
\text { elimination activities } \\
\text { in residential areas } \\
\text { should be left entirely } \\
\text { to the government }\end{array}$ & $7(4.6)$ & $12(7.9)$ & $70(46.1)$ & $63(41.4)$ & \\
\hline & & & 20 & $\begin{array}{l}\text { I do not think I played } \\
\text { an important role in } \\
\text { controlling dengue } \\
\text { fever }\end{array}$ & $6(3.9)$ & $13(8.6)$ & $69(45.4)$ & $64(42.1)$ & \\
\hline & & & 21 & $\begin{array}{l}\text { I have sufficient } \\
\text { knowledge on dengue } \\
\text { fever }\end{array}$ & $10(6.6)$ & $\begin{array}{l}88 \\
(57.9)\end{array}$ & 47 (30.9) & 7 (4.6) & \\
\hline & & & 22 & $\begin{array}{l}\text { I do not know what } \\
\text { information needs to } \\
\text { be delivered to my } \\
\text { family members about } \\
\text { dengue }\end{array}$ & $3(2.0)$ & $\begin{array}{l}18 \\
(11.8)\end{array}$ & $80(52.6)$ & $51(33.6)$ & \\
\hline \multicolumn{10}{|c|}{ Perceived barrier } \\
\hline $\begin{array}{l}\text { Unsatisfactory } \\
\text { attitude }\end{array}$ & 145 & 95.4 & 17 & $\begin{array}{l}\text { I felt that the } \\
\text { government measures } \\
\text { in controlling/ } \\
\text { preventing dengue is } \\
\text { not effective }\end{array}$ & $10(6.6)$ & $\begin{array}{l}23 \\
(15.1)\end{array}$ & $77(50.7)$ & $42(27.6)$ & \multirow{5}{*}{0.737} \\
\hline \multirow[t]{4}{*}{$\begin{array}{l}\text { Satisfactory } \\
\text { attitude }\end{array}$} & 7 & 4.6 & 23 & $\begin{array}{l}\text { I did not allow fogging } \\
\text { in house as it is } \\
\text { hazardous to health }\end{array}$ & $9(5.9)$ & $11(7.2)$ & $61(40.1)$ & 71(46.7) & \\
\hline & & & 24 & $\begin{array}{l}\text { Use of larvicide in the } \\
\text { water can cause the } \\
\text { water to become toxic }\end{array}$ & $7(4.6)$ & $\begin{array}{l}17 \\
(11.2)\end{array}$ & $51(33.6)$ & $77(50.7)$ & \\
\hline & & & 25 & $\begin{array}{l}\text { My family members } \\
\text { are not interested } \\
\text { when I shared } \\
\text { information about } \\
\text { dengue }\end{array}$ & $2(1.3)$ & $14(9.2)$ & $70(46.1)$ & $66(43.4)$ & \\
\hline & & & 26 & $\begin{array}{l}\text { I have other important } \\
\text { tasks causes me } \\
\text { unable to convey } \\
\text { information about } \\
\text { dengue to my family }\end{array}$ & $4(2.6)$ & $11(7.2)$ & $57(37.5)$ & $80(52.6)$ & \\
\hline \multicolumn{10}{|c|}{ Perceived benefit } \\
\hline $\begin{array}{l}\text { Unsatisfactory } \\
\text { attitude }\end{array}$ & 11 & 7.2 & 18 & $\begin{array}{l}\text { I will ensure my house } \\
\text { area is free from } \\
\text { breeding spot to avoid } \\
\text { being penalized by the } \\
\text { health authority }\end{array}$ & $91(59.9)$ & $\begin{array}{l}56 \\
(36.8)\end{array}$ & $4(2.6)$ & $1(0.7)$ & \multirow[t]{2}{*}{0.783} \\
\hline $\begin{array}{l}\text { Satisfactory } \\
\text { attitude }\end{array}$ & 141 & 92.8 & 27 & $\begin{array}{l}\text { My participation in } \\
\text { control/prevention } \\
\text { activities can help in } \\
\text { controlling dengue }\end{array}$ & $58(38.2)$ & $\begin{array}{l}88 \\
(57.9)\end{array}$ & $4(2.6)$ & $2(1.3)$ & \\
\hline
\end{tabular}




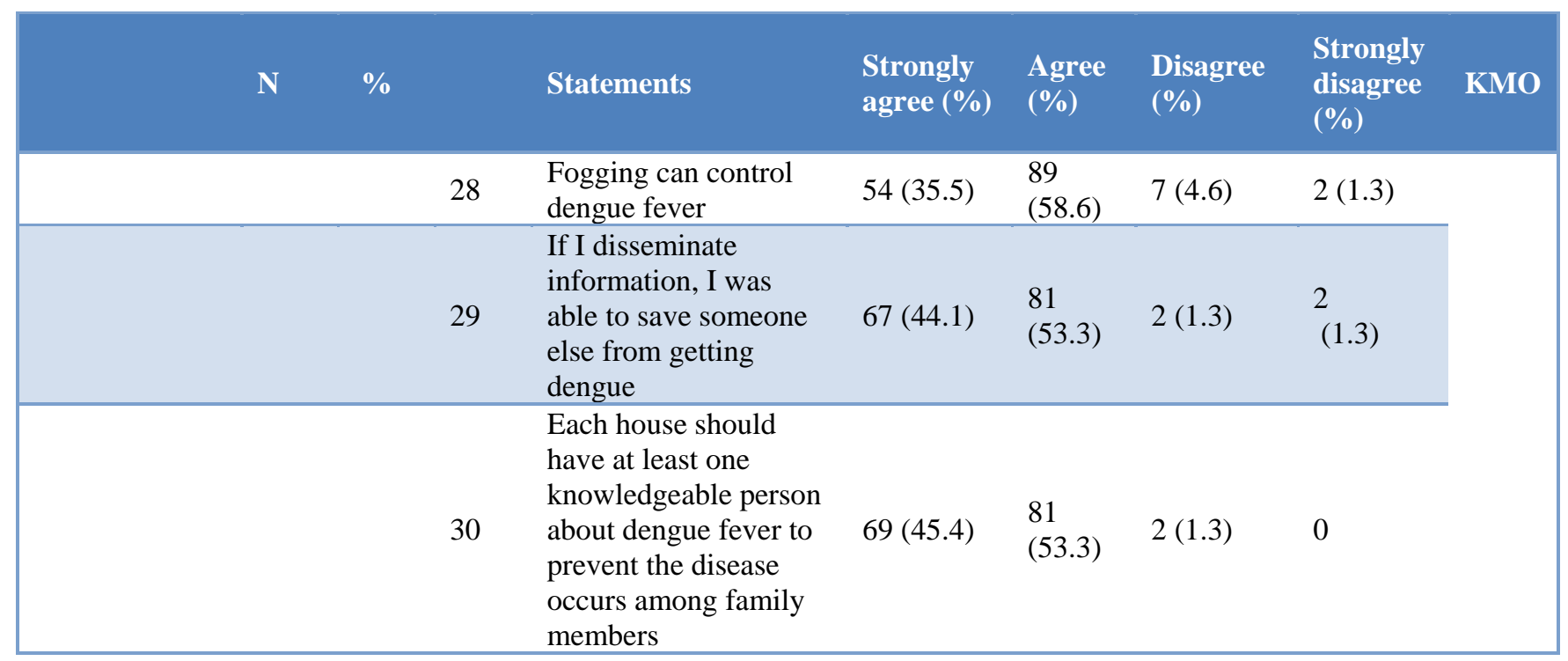

Table 5: Correlation between knowledge, attitude and practices of dengue prevention.

\begin{tabular}{|llll|}
\hline Variables & r-value & P value & Interpretation \\
\hline Knowledge vs attitude & +0.071 & 0.386 & Small positive correlation \\
\hline Attitude vs practice & +0.093 & 0.254 & Small positive correlation \\
\hline Knowledge vs practice & +0.179 & 0.027 & Small positive correlation \\
\hline
\end{tabular}

Table 6: Comparison knowledge, attitude and practice of dengue between groups of gender, age, race, marital status, educational background, residential property and history of dengue $(n=152)$.

\begin{tabular}{|c|c|c|c|c|c|c|c|c|c|}
\hline & & & & Knowledge & & Attitude & & Practice & \\
\hline S. no. & Variables & $\mathrm{N}$ & $\%$ & $\begin{array}{l}\text { Median } \\
\text { (IQR) }\end{array}$ & $P$ value & $\begin{array}{l}\text { Median } \\
\text { (IQR) }\end{array}$ & $\mathrm{P}$ value & $\begin{array}{l}\text { Median } \\
\text { (IQR) }\end{array}$ & $P$ value \\
\hline \multirow[t]{3}{*}{1} & Gender & & & & 0.316 & & 0.743 & & 0.122 \\
\hline & Male & 114 & 75.0 & $34(8)$ & & $83.5(7.25)$ & & $10(2)$ & \\
\hline & Female & 38 & 25.0 & $32(7.25)$ & & $84.47(8)$ & & $9(3)$ & \\
\hline \multirow[t]{4}{*}{2} & Age (in years) & & & & 0.865 & & 0.510 & & 0.300 \\
\hline & $18-30$ & 8 & 5.2 & $32(12.75)$ & & $84.5(9.5)$ & & $9(1.75)$ & \\
\hline & $31-50$ & 23 & 15.2 & $34(5)$ & & $83(7)$ & & $9(1)$ & \\
\hline & $>51$ & 121 & 79.6 & $33(9)$ & & $83(7.5)$ & & $10(2)$ & \\
\hline \multirow[t]{4}{*}{3} & Marital status & & & & 0.826 & & 0.764 & & 0.803 \\
\hline & Single & 8 & 5.3 & $35(16)$ & & $84(7.25)$ & & $9(2.5)$ & \\
\hline & Married & 141 & 92.8 & $33(9)$ & & $83(7.5)$ & & $10(2)$ & \\
\hline & Widower/widower & 3 & 2.0 & 32 & & 83 & & 9 & \\
\hline \multirow[t]{7}{*}{4} & Educational attained & & & & 0.092 & & 0.862 & & 0.993 \\
\hline & No formal education & 0 & 0 & - & & - & & - & \\
\hline & Primary & 0 & 0 & - & & - & & - & \\
\hline & Secondary & 21 & 14.0 & $32(9.5)$ & & $84(9)$ & & $10(2)$ & \\
\hline & Diploma & 34 & 22.7 & $29(15)$ & & $83.5(11)$ & & $10(2)$ & \\
\hline & Degree & 78 & 52.0 & $34(7)$ & & $83(5)$ & & $10(2)$ & \\
\hline & Others & 17 & 11.3 & $33(8)$ & & $86(8)$ & & $10(2)$ & \\
\hline \multirow[t]{3}{*}{5} & Residential property & & & & 0.200 & & 0.035 & & 0.393 \\
\hline & Owner & 136 & 89.5 & $34(8)$ & & $83(7)$ & & $10(2)$ & \\
\hline & Rent & 16 & 10.5 & $31(13.25)$ & & $85.5(8.75)$ & & $10(2.75)$ & \\
\hline \multirow[t]{3}{*}{6} & History with dengue fe & ver & & & 0.716 & & 0.357 & & 0.500 \\
\hline & Yes & 45 & 29.6 & $34(10.5)$ & & $82(8)$ & & $10(2)$ & \\
\hline & No & 107 & 70.4 & $33(9)$ & & $84(8)$ & & $10(2)$ & \\
\hline
\end{tabular}




\section{Attitude towards dengue}

The attitudes of the respondents were assessed using a set of questions regarding dengue and Aedes mosquito prevention (Table 4). These questions were based from Health Believed model (HBM) with its fundamental perceives. Through perceived severity, all of the respondents strongly agreed and agreed that dengue is a dangerous and serious illness. The respondents would get very distressed when a member of the household is having continuous fever with strongly agreed at $65.1 \%$ $(n=99)$. 97.4\% $(n=182)$ respondents strongly agreed and agreed that they are still concern about getting dengue although all the medical facilities are available within their surroundings. However, another interesting findings from these respondents, there are $5.3 \%(n=8)$ respondents considered dengue would not causing death.

On the perceived susceptibility, $57.9 \%(n=88)$ believe that they are all vulnerable to dengue. They realized that Aedes mosquito bites will eventually make them sick with dengue although they are healthy and well (38.2\%, $\mathrm{n}=58)$. They are worry if they found any standing water surrounding their premises $(77.6 \%, \mathrm{n}=118)$ while they are 4 respondents $(2.6 \%)$ who did not bother if there is standing water surrounding their premises. Following the perceived susceptibility, the cues for action was asked and $49.3 \%(n=75)$ of the respondents are ready to take action against the Aedes mosquito.

Interestingly, on the attitude for self-efficacy, $31.6 \%$ $(n=51)$ of the respondents declared said they know what kind of information they would pass on to their family member/s as $57.9 \% \quad(n=88)$ of them have sufficient knowledge on dengue and Aedes mosquito. 60.5\% agreed that they will ensure there will be no breeding sites in their premises. $41.4 \%(n=63)$ said that they are the one who responsible for the breeding sites surrounding their premises and only $4.6 \% \quad(n=7)$ respondents had a consensus that the government has the prime responsibility to control mosquito breeding. $42.1 \%$ $(n=64)$ respondents strongly agreed and agreed that they had played their roles in controlling dengue fever in their areas.

As for the perceived barrier, the overall score for attitude is considered weak negative although the respondents as individually had positive attitude. Only $2.6 \% \quad(n=4)$ perceived themselves as having other important tasks causes them unable to convey information about dengue to their family. For the perceived benefit, 58.6\% agreed that they will benefitted very much once practiced all the preventive measure. They also agreed that by disseminating the knowledge on dengue and Aedes mosquito could help saving somebody from dengue $(53.3 \%, \mathrm{n}=81)$. In doing so, at least one member from each household should be knowledgeable in dengue and Aedes mosquito $(53.3 \%, \mathrm{n}=81)$.

\section{Practice of dengue control}

Figure 2 lists the distribution of preventive practices in our sample. In this study, the majority of the participants $(78.9 \%, \mathrm{n}=120)$ had a total dengue prevention practice score in the range of $75-100$. Out of 187 respondents, $148(97.4 \%)$ throw waste in tied plastic trash and closed the trash lid properly. The next highest practice is covering the stored water tightly $(94.7 \%, \mathrm{n}=144)$ followed by removing all the damaged equipment that can stored water $(93.4 \%, n=142$ The respondent second least preventive measure is the usage of the larvicide $(55.3 \%, \mathrm{n}=84)$. The least preventive method is brushing and scrub the container weekly $(48.2 \%, \mathrm{n}=128)$.

In this study, there is a positive significant correlation between knowledge and practices of dengue (Table 5). The respondents in Bandar Baru Bangi, have a significance difference between the owner of the house and the tenant regarding the attitude on dengue (Table 6). Further analysis through Mann-Whitney $U$ test has showcased that the self-efficacy has a significant difference between the house ownership $(\mathrm{p}=0.010$, $\mathrm{p}<0.05, \mathrm{Md}$ of house owner=11, IQR=2.0, Md of rent=13, IQR=1.0). A Mann Whitney test indicated that the self-efficacy of female (Median=12, $n=38$ ) respondents were significantly higher than male (median=11, $\mathrm{n}=114$ ) respondents, $\mathrm{U}=1653.50, \mathrm{z}=-2.223$, $\mathrm{p}=0.026$.

\section{DISCUSSION}

Our study was try to find to evaluate public knowledge, attitudes and practices related to dengue infection among 152 respondents in Bandar Baru Bangi, Malaysia. Our study finds that only $52 \%$ of the respondents have sufficient knowledge on dengue and Aedes mosquito but $15.1 \%$ and $78.9 \%$ have satisfactory attitude and practices against dengue, respectively (Figure 3 ). To the best of our knowledge, this is one of utmost important finding that suggest insufficient level of knowledge could somehow appended with good attitudes and practices directed at reducing the prevalence of the disease. This could perhaps be due to them who had been living in the 'hotspot' area and seen neighbors and friends who were badly affected by the dengue fever. However, this finding is inconsistent to that observed in Puerto Rico where those with a previous dengue diagnosis were more knowledgeable about dengue and concerned about others getting dengue. ${ }^{9}$

Our study revealed that almost all respondents to the questionnaires are familiar with dengue disease. Despite the revolution of smart phone and the availability of wireless internet, television/radio was reported as the most common source of information. This is similar to previous studies whereby television was cited to have a major role in disseminating information about dengue. ${ }^{7,8,10,11}$ Again, the role of mass media is crucial in getting the message across to the public. ${ }^{12}$ Another 
interesting fact to be highlighted through this study is the health personnel is the least source of information for dengue. This finding was inconsistent with another study conducted in Thailand which found that health personnel were the main source of dengue fever information. ${ }^{13}$

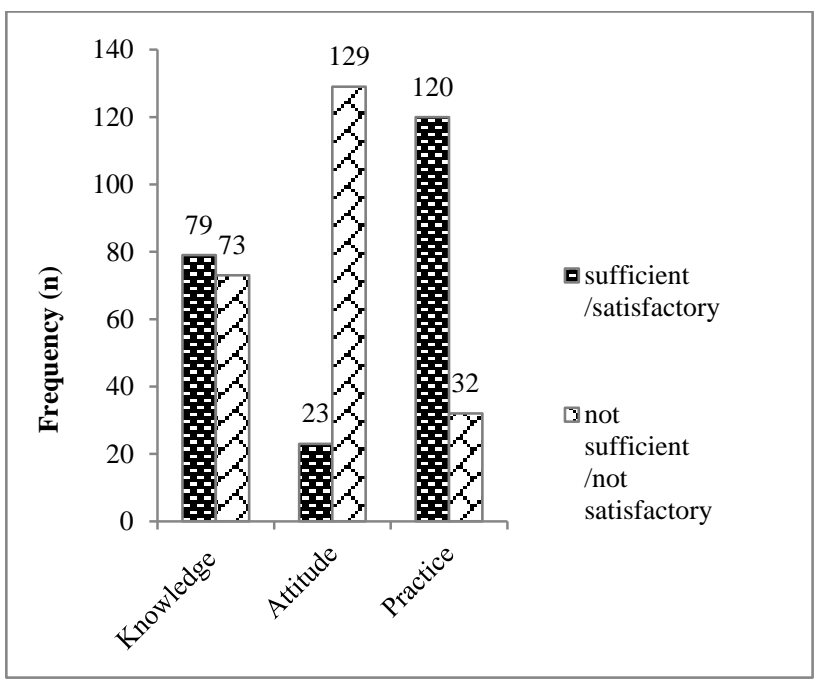

Figure 3: Summarized levels of knowledge, attitude and prevention practices of the respondents in Bandar Baru Bangi, Selangor $(n=152)$.

The majority of respondents are aware of symptoms of dengue and able to recognize high fever, joint pain, rash and headache as the main symptoms. This was probably due to the educational message from the mass media that the primary symptom of dengue is a high fever of sudden onset with joint pain. Their ability to recognize the signs and symptoms of dengue was important for them to seek early treatment. The respondents showed considerably good knowledge about the symptoms, with fever being correctly accounted as the most common. Adequate knowledge on dengue symptoms has been reported in similar studies done in Pakistan, India and Brazil. ${ }^{14-16}$

However, when it comes to the knowledge on the Aedes mosquito as the dengue vector, this is where many of the respondents are lacking of. Almost half (48.0\%) of the respondents didn't know that the life cycle of the Aedes mosquito is 7 days and moreover, $25.7 \%$ of the respondents couldn't correctly answer the average flight range of the vectors is 200 meter. Knowledge on the life cycle justifies why eliminating of the breeding places need to be routinely perform weekly. While knowledge on the flight range explain why the fogging activity was performed 200 meter radius from the patient house. Only $65.8 \%$ correctly replied that the peak biting time was during the dusk and dawn of the day. This finding was inconsistent with the findings of some previous studies, which indicated the majority of respondents knew mosquitoes transmitting dengue might bite either at sunrise or sunset. ${ }^{14,17}$ Despite that insufficient knowledge on Aedes mosquito, the respondents showed sufficient knowledge on its physical characteristic whereby $81.6 \%$ were aware that the Aedes mosquito has black and white stripes on its legs and body.

The respondents has adequate knowledge related to the mosquito and dengue disease on isolated aspects, but the overall prevalence of 'sufficient knowledge' based on our criteria is moderate. However, based on the questionnaire, the claimed they practiced the preventive measures against dengue vectors. Furthermore, a high portion of participants $(98.7 \%)$ believed that eliminating the Aedes breeding places was their responsibilities (i.e., proportion with high agreement instead that controlling dengue was the responsibility of the government)

Previous research has shown that the level of education has a significant impact on knowledge related to dengue. ${ }^{14}$ Therefore, dengue prevention and control campaigns should engage urban middle to higher income earners who mostly work away from home to allocate their time to carry out dengue prevention practices at home and in their neighborhoods. ${ }^{18}$ That study also shown that, skilled workers were also less likely to practice dengue prevention compared to those unemployed. This could be because those who are unemployed, usually housewives, spend a lot of time at home and are most likely to carry out household cleaning activities. This clarified the reason of having high selfefficacy among female respondents in this study.

The current study findings were consistent with the theory of health belief model where self-efficacy had the major predictive power than the other predictor variables in predicting intention to conduct the dengue preventive measure. The findings from our study provide a base for creating educational and health messages for health behavioural change intervention based on the HBM constructs. HBM is a psychological model that attempts to explain and predict health behaviors by focusing on the attitudes and beliefs of individuals. These concepts were proposed as accounting for people's "readiness to act." An added concept, cues to action, would activate that readiness and stimulate overt behavior. A recent addition to the HBM is the concept of self-efficacy, or one's confidence in the ability to successfully perform an action. $^{19}$

In this study, the sufficient knowledge has increased the strength of people's confidence in performing dengue prevention activity especially among the female respondents. This is similar with a study in Terengganu where simple message was delivered through engagement with simple enacted interim behavior that has enhance self-efficacy. ${ }^{20}$ The outcome of this study indicated that increase in knowledge will increase the practice against dengue. This is supported by the previous study in Havana, where the knowledge of dengue has a direct positive effect upon preventive practices. $^{21}$

This study found that the most commonly practiced preventive measures used by the respondents were throw 
waste in tied plastic trash and closed the trash lid properly, covering the stored water tightly, followed by frequently changed the stored water. These findings were consistent with those reported by previous studies. ${ }^{11,14,22}$ The respondent also declared they had participated at least once in any clean-up campaign against dengue. Some of the respondent least preventive measure is the usage of the common larvicide $(55.3 \%, \mathrm{n}=84)$. This study consistent with the findings from a previous study from Thailand where they found that temephos was not the preferred choice as the respondents dislike drinking water containing temephos sand or granules. ${ }^{12,23}$

A study in residential area of Kuala Lumpur has concluded that there is a need to strengthen health promotion activities to increase the knowledge as part of the strategy to control dengue outbreaks. ${ }^{10}$ The media are important sources to convey health messages to the public; thus the research and development of educational strategies designed to improve behavior and practices of effective control measures among population is essential. ${ }^{7}$ Prevention programs are more effective if the knowledge and vector control practices of the population are understood and applied in the main stream of intervention activities. $^{13,24,25}$ Therefore, it is essential to enhance knowledge about the community's perception about dengue as well as their vector control practices before and after the launching of any community-based vector control programs.

\section{Limitation}

This study has some limitations. Firstly it is expected to be prone for the limitation of cross-sectional survey (temporal relationship). Our findings must be interpreted in the light of several potential limitations the most apparent of which may be the fact that a cross-sectional survey assesses relationships based on one point in time and it does not account for the dynamics of relationships between variables evaluated. Secondly, it is possible that since the survey was interviewer based use of questionnaires, some participants would provide socially desirable responses to some questions. Thirdly, the small sample size may have limited our ability to detect associations that were small and moderate in magnitude and yielded estimates that lacked precision.

\section{CONCLUSION}

As the conclusion, adult population of Bandar Baru Bangi has adequate knowledge related to dengue. Yet, the overall prevalence of 'sufficient knowledge' based on our criteria is moderate. This interpreted into poor attitude yet perplexed as the respondents has good preventive practices against the disease. Possibly, the critical dengue situation in their areas compelled them to conduct the preventive practices.

\section{ACKNOWLEDGEMENTS}

Special thanks to Ministry of Science, Technology and Innovation of Malaysia for the funding under the flagship project code FP0914D0026-2 (DSTIN). We thank the residents of Section 3 and section 8 of Bandar Baru Bangi, Selangor, Malaysia for participating in the KAP survey. The collaboration with the staffs of Health District Office, MPKJ, resident associations and the local public health volunteers is greatly appreciated. We also thank National University of Malaysia for the financial support (UKM-Komuniti-2014) and InnoBiologics Sdn Bhd and EntoGenex Industries Sdn. Bhd. for the invaluable assistance throughout the study.

Funding: No funding sources

Conflict of interest: None declared

Ethical approval: The study was approved by the Institutional Ethics Committee

\section{REFERENCES}

1. World Health Organization. Dengue and severe dengue, 2016. Available at: http://www.who.int/ mediacentre/factsheets/fs117/en/. Accessed on 20 August 2016.

2. Samir B, Peter WG, Oliver JB, Jane PM, Andrew $\mathrm{WF}$, et al. The global distribution and burden of dengue. Nature. 2013;496(7446):504-7.

3. World Health Organization. A global belief on vector-borne disease. Geneva, Switzeland: WHO, 2014.

4. Ministry of Health Malaysia. Press statement "Ops Mega" Achievement, 2014.

5. Rahman AA, Zainuddin H, Minhat HS, Juni MH, Mazeli MI. Community perception towards dengue and dengue prevention program among residences of a rural settlement in Jempol, Negeri Sembilan. Int J Public Health Clin Sci. 2014;1:13-26.

6. Dhimal M, Aryal KK, Dhimal ML, Gautam I, Singh SP, Bhusal CL, et al. Knowledge, attitude, and pratice regarding dengue fever among the healthy population on highland and lowland communities in Central Nepal. PLoS One. 2014;9(7):e102028.

7. Shuaib F, Todd D, Campbell-Stennett D, Ehiri J, Jolly PE. Knowledge, attitudes and practices regarding dengue infection in Westmoreland, Jamaica. West Indian Med J. 2010;59:139.

8. Wan Rozita W, Yap B, Veronica S, Mohammad A, Lim K. Knowledge, attitude and practice (KAP) survey on dengue fever in an urban Malay residential area in Kuala Lumpur. Malays J Public Health Med. 2006;6:62-7.

9. Acharya A, Goswami K, Srinath S, Goswami A. Awareness about dengue syndrome and related preventive practices amongst residents of an urban resettlement colony of South Delhi. J Vector Borne Dis. 2005;42:122-7.

10. Ibrahim NKR, Al-Bar A, Kordey M, Al-Fakeeh A. Knowledge, attitudes, and practices relating to 
Dengue fever among females in Jeddah high schools. J Infect Public Health. 2009;2:30-40.

11. Kittigul L, Suankeow K, Sujirarat D, Yoksan S. Dengue hemorrhagic fever: knowledge, attitude and practice in Ang Thong Province, Thailand. Southern Asian J Trop Med Public Health. 2003;34(2):38592.

12. Nalongsack S, Yoshida Y, Morita M, Sosouphanh K, Sakamoto J. Knowledge, attitude and practice regarding dengue among people in Pakse, Laos Nagoya. J Med Sci. 2009;71:29-37.

13. Itrat A, Khan A, Javaid S, Kamal M, Khan H, Javed $\mathrm{S}$, et al. Knowledge, awareness and practices regarding dengue fever among the adult population of dengue hit cosmopolitan. PLoS One. 2008;3:e2620.

14. Van Benthem BHB, Khantikul N, Panart K, Kessels PJ, Somboon P, Oskam L. Knowledge and use of prevention measures related to dengue in Northern Thailand. Trop Med Int Health. 2002;7(11):9931000 .

15. World Health Organization. Dengue Haemorrhagic Fever: Diagnosis, Treatment, Prevention and Control. 2nd Ed. Geneva: WHO, 1997. Available at: http://www.who.int/csr/resources/publications/deng ue/Denguepublication/en/. Accessed on 20 August 2016.

16. Degallier N, Vilarinhos PTR, Carvalho MSL, Knox MB, Caetano JJr. People's knowledge and practice about dengue, its vectors, and control means in Brasilia (DF), Brazil: Its relevance with entomological factors. J Am Mosq Control Assoc. 2000;16(2):114-23.

17. Wong LP, Shakir SMM, Atefi N, Abu Bakar S. Factors affecting dengue prevention practices: Nationwide survey of the Malaysian Public. PLoS One. 2015;10:0122890.

18. Sutton S. A critical review of the Transtheoretical Model Applied to Smoking Cessation. In: Norman P, Abraham C, Conner M, eds. Understanding and changing health behaviour: From health beliefs to self-regulation. London: Harwood Academic Publishers; 2000:207-25.
19. Lennon JL. The use of the health belief model in dengue health education. Dengue Bull. 2005;29:217-9.

20. Affendi I, Loke YK, Smith JR, Papageorgio A, Hunter PR. Mediational Effects of Self Efficacy Dimensions In The Relationship Between Knowledge Of Dengue And Dengue Preventive Behavior With Respect To Control Of Dengue Outbreaks: A Structural Equation Model Of A Cross Sectional Survey. PLoS Negl Trop Dis. 2013;7(9):110.

21. Castro M, Sanche ZL, Perez D, Sebrango C, Shkedy Z, Van der Stuyft P. The relationship between Economic Status, Knowledge on Dengue, Risk Perception and Practices. PLOS One. 2013;8(2):1-8.

22. Snehalatha K, Ramaiah K, Kumar KV, Das P. The mosquito problem and type and costs of personal protection measures used in rural and urban communities in Pondicherry region, South India. Acta tropica. 2003;88:3-9.

23. Swaddiwudhipong W, Chaovakiratipong C, Nguntra P, Koonchote S, Khumklam P, Lerdlukanavonge P. Effect of health education on community participation in control of dengue hemorrhagic fever in an urban area of Thailand. Southeast Asian J Trop Med Public Health. 1992;23:200-6.

24. Leontsini E, Gil E, Kendall C, Clark GG. Effect of a community-based Aedes aegypti control programmed on mosquito larval production sites in El Progreso, Honduras. Trans R Soc Trop Med Hyg. 1993;87:267-71.

25. Lloyd LS, Winch P, Ortega-Canto J, Kendall C. The design of a community-based health education intervention for the control of Aedes aegypti. Am J Trop Med Hyg. 1994;50:401-11.

Cite this article as: Othman H, Zaini ZI, Karim N, Rashid NAA, Abas MBH, Sahani M, et al. Applying health belief model for the assessment of community knowledge, attitude and prevention practices following a dengue epidemic in a township in Selangor, Malaysia. Int J Community Med Public Health 2019;6:958-70. 